\title{
La responsabilità del primario nell'attività in équipe
}

\author{
- Alessandro Buzzoni ${ }^{1}$ \\ 1 Avvocato, Rimini
}

\begin{abstract}
Teamwork is now a fact of life for physicians working in hospitals and other institutional settings. When a case of malpractice occurs, it is necessary to define the extent of liability of each member of the team. In this article, the Author analyzes specifically the role of the head physician. Juridical and medico-legal aspects are evaluated on the basis of civil law and specific regulations now in force.
\end{abstract}

Keywords: teamwork, head physician, medical liability

Teamwork and medical liability of the head physician

Pratica Medica \& Aspetti Legali 2008; 2(4): 199-202

\section{INTRODUZIONE}

Lattività medico-chirurgica in équipe è contraddistinta dalla partecipazione e collaborazione tra più medici e Sanitari, che interagiscono per il raggiungimento di un obiettivo comune [1].

Sempre più spesso, infatti, il corretto inquadramento di una patologia richiede uno screening complesso, formato da varie fasi diagnostiche, esami clinici e strumentali. La sua gestione implica quindi la collaborazione tra diverse figure professionali, quali radiologi, medici di laboratorio, chirurghi e anestesisti. L'attività in équipe è stata foriera nel tempo di importanti problematiche di natura giurisprudenziale, riguardanti l'esatta identificazione del dovere di diligenza ascrivibile ad ogni membro del gruppo di lavoro. In particolare, in seguito alla continua espansione delle specializzazioni in campo medico, si è manifestata l'esigenza di fronteggiare gli aspetti giuridici dei cosiddetti "obblighi divisi”, caratterizzati appunto da una suddivisione degli interventi medici nell'ambito di un medesimo percorso diagnostico e terapeutico [1].

In questo articolo sarà in particolare esaminata la responsabilità del primario.

\section{DESCRIZIONE DEL CASO}

In un procedimento relativo a un reato di omicidio colposo di un neonato, la Corte ha riconosciuto la responsabilità del primario del reparto ostetrico-ginecologico che, unitamente all'assistente che aveva in cura la donna in stato di gravidanza, aveva deciso di procedere comunque nel travaglio senza ricorrere al parto cesareo, nonostante fossero state evidenziate alcune anomalie nella paziente.

Il primario, inoltre, aveva abbandonato la sala prima del parto e aveva lasciato la donna nelle mani dell'assistente che, per assecondare l'espulsione del feto, aveva impiegato una ventosa anziché il forcipe, che avrebbe invece alleviato le sofferenze della gestante e accelerato il parto.

Del conseguente evento lesivo era stato dunque chiamato a rispondere anche il primario: questi, infatti, aveva fatto affidamento sulla correttezza delle manovre effettuate dall'assistente, che erano poi invece risultate errate. La Corte, inoltre, lo aveva giudicato responsabile di aver lasciato la sala parto senza esercitare l'attività di controllo che rientrava nei suoi obblighi [2]. 


\section{DISCUSSIONE}

La dottrina più recente ha sostenuto che, nel caso di interventi in cui siano coinvolti più terapeuti, coloro che si trovano in posizione di vertice, avendo la direzione e il potere di coordinamento dell'attività medica dei propri assistenti, sono obbligati al controllo dell'operato altrui [3-6].

Pertanto il capo dell'équipe, oltre a dovere attuare con cura, diligenza e perizia le funzioni specifiche a lui spettanti, è tenuto a coordinare l'attività dei propri collaboratori e verificare la correttezza nell'esecuzione dei compiti loro affidati. Di conseguenza, nel caso in cui si verifichi un evento lesivo determinato dalla condotta di uno dei membri del team, il capo sarà considerato responsabile per omesso o insufficiente controllo del suo operato. In questa linea di giudizio rientra anche la sentenza sopra riportata: il capo dell'équipe medica è infatti ritenuto responsabile per gli esiti infausti dei trattamenti chirurgici effettuati dal gruppo di lavoro da lui coordinato e diretto.

In tal senso è possibile citare altre due sentenze:

- in una sentenza della Corte è stata riconosciuta la responsabilità di un anestesista per aver omesso di vigilare sull'operato di un suo sottoposto, da lui incaricato della sostituzione di un flacone di sangue, poi risultato incompatibile con il gruppo sanguigno del paziente che, in seguito all'errore, era deceduto [7];

- in una vicenda relativa a un intervento chirurgico nel quale, per errore, era stato dimenticato uno strumento chirurgico nell'addome del paziente, la Cassazione ha puntualizzato che doveva comunque ritenersi esclusa l'operatività del principio dell'affidamento nei rapporti intercorrenti tra personale medico e paramedico, specie quando quest'ultimo viene incaricato di eseguire attività di sostegno e di assistenza in genere del Sanitario, sul quale grava pertanto l'obbligo di un continuo e pedissequo controllo del suo operato [8].

\section{LA POSIZIONE DEL PRIMARIO}

La figura del primario ricopre l'obbligo di garanzia nei confronti dei pazienti; pertanto è ritenuto in ogni caso responsabile per gli eventi lesivi determinati dalle condotte poste in essere dai componenti del suo reparto. Egli, infatti, rivestendo una posizione apicale di garanzia, è tenuto a specifici obblighi di verifica e di sorveglianza verso tutti i membri della propria équipe, impartendo direttive e controllando pedissequamente l'operato di tutti i suoi assistenti. In particolare le sue responsabilità comprendono, secondo le basi legislative di riferimento (Tabella I), obblighi di organizzazione, di didattica e di valutazione nei confronti del personale sottoposto e di tutti i suoi assistenti. Nel caso di attività medica multidisciplinare, la normativa individua la cosiddetta regola dell'affidamento reciproco. Secondo tale principio, i vari professionisti coinvolti devono ritenersi responsabili per gli eventuali errori imputabili ai propri colleghi nel caso in cui, utilizzando l'ordinaria diligenza, avrebbero potuto individuarli e segnalarli al responsabile del gruppo.

Detto altrimenti, la responsabilità viene inquadrata come una forma di "aspettativa parziale" dei vari membri del gruppo medico, che trova il suo limite di applicabilità nella circostanza dell'imprevedibilità dell'evento lesivo oggetto dell'accertamento e della inevitabilità delle sue conseguenze morbose [9].

Ed è proprio in tale ambito che deve essere analizzata la posizione del responsabile del gruppo operativo: egli ha infatti un compito fondamentale di sorveglianza e di controllo sull'operato dei suoi sottoposti, nascente da una posizione giuridica di garanzia, che gli conferisce i poteri di controllo e di coordinamento del lavoro d'équipe. Un altro

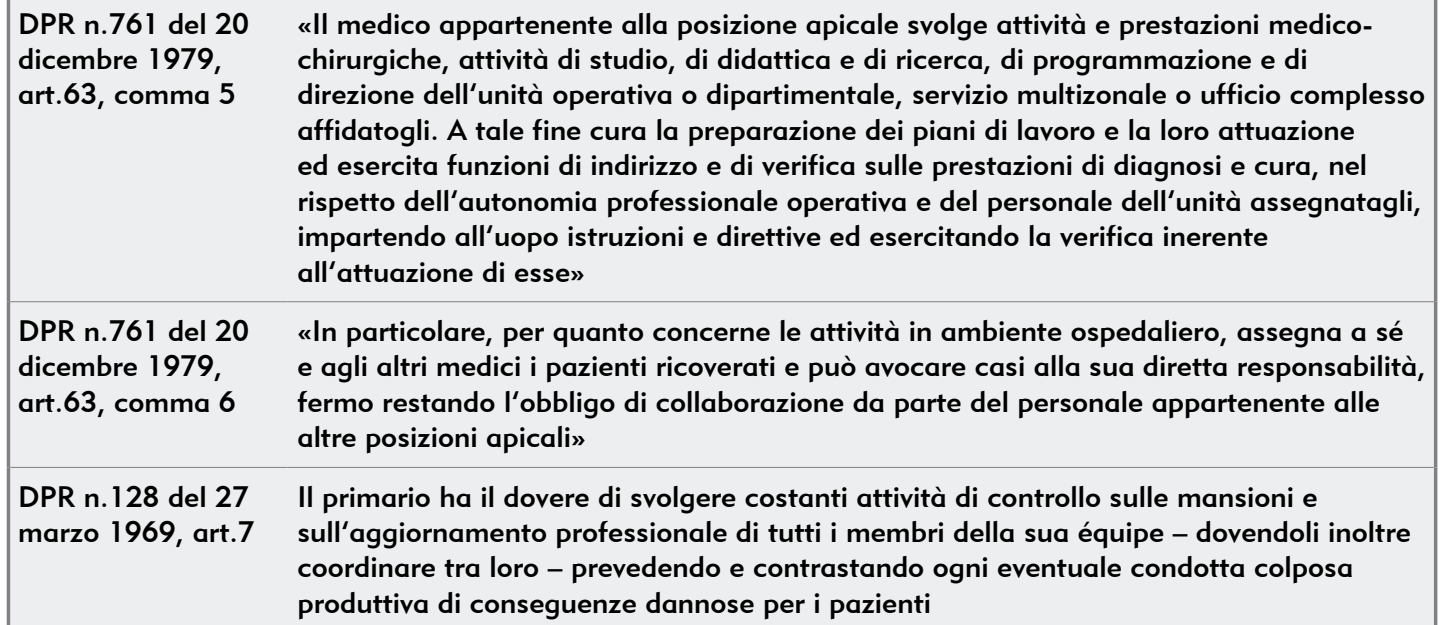

DPR n.761 del 20 «In particolare, per quanto concerne le attività in ambiente ospedaliero, assegna a sé dicembre 1979, e agli altri medici i pazienti ricoverati e può avocare casi alla sua diretta responsabilità, art.63, comma 6 fermo restando l'obbligo di collaborazione da parte del personale appartenente alle altre posizioni apicali»

DPR n.128 del 27 II primario ha il dovere di svolgere costanti attività di controllo sulle mansioni e marzo 1969, art.7 sull'aggiornamento professionale di tutti i membri della sua équipe - dovendoli inoltre coordinare tra loro - prevedendo e contrastando ogni eventuale condotta colposa produttiva di conseguenze dannose per i pazienti

Tabella I. Normativa di riferimento 
caso riguarda il ricovero di un paziente per pancreatite acuta, successivamente aggravatasi in una occlusione intestinale immediatamente riconosciuta dall'aiuto primario, che aveva informato della circostanza i familiari del paziente, ma non anche il suo superiore gerarchico, rendendosi anzi partecipe del comportamento approssimativo di quest'ultimo che aveva considerato l'intervento assolutamente urgente (in seguito ad esso il paziente era deceduto). In questo caso la Corte di Cassazione ha ritenuto configurata la responsabilità concorsuale del primario e dell'aiuto primario $[10,11]$. La responsabilità penale del capo-équipe per gli eventuali esiti infausti dell'intervento operatorio discenderà dunque dall'aver questi omesso di intervenire adeguatamente per limitare o eliminare le conseguenze lesive generate dal comportamento negligente o imprudente dei suoi sottoposti.

Anche le posizioni della dottrina si sono uniformate a tale inquadramento, affermando che: "così interpretati i concetti di dovere e controllo e di dovere di coordinamento, la responsabilità a titolo di colpa del capo-équipe per esito infausto ricollegabile a negligenza o imprudenza altrui, si profilerà se egli abbia tenuto un contegno omissivo che avrebbe dovuto indurlo ad intervenire con le iniziative del caso (azioni colpose già in atto, precarie condizioni psico-fisiche di qualche membro dell'équipe, assenza di qualche collaboratore, ecc.) e alle quali sia causalmente riconducibile l'esito infausto verificatosi» [9].

Nella giurisprudenza è frequente trovare affermazioni di responsabilità del primario per omesso 0 inadeguato esercizio dell'obbligo di direzione e controllo dell'operato dei suoi sottoposti. Alcuni esempi in tal senso sono:

- in un caso di omicidio colposo in seguito a un sinistro stradale, la Corte ha optato non soltanto per la responsabilità dei Sanitari facenti parte dell'équipe medica, per non essere riusciti ad identificare in maniera compiuta la sintomatologia del paziente, omettendo in tal modo di porre in essere i rimedi più elementari e necessari, facenti parte del patrimonio cognitivo di qualsiasi medico anche non specialistico, ma anche del primario, il quale non aveva adottato le necessarie funzioni di indirizzo, verifica e direzione dell'attività dei suoi subalterni, per l'esecuzione delle migliori e più opportune scelte terapeutiche del caso [12];

- in un caso di morte per shock anafilattico di un paziente al quale era stato somministrato dal medico di guardia del Pronto Soccorso una dose di un farmaco, senza che questi avesse accertato eventuali allergie allo stesso, la Corte ha riconosciuto la responsabilità del primario rispetto agli eventi lesivi cagionati dal comportamento colposo posto in essere dai suoi subalterni [13].
In quest'ultima sentenza citata, oltre alla responsabilità concorrente del medico di guardia, la Corte ha così riconosciuto la pari responsabilità del primario, presente in Pronto Soccorso, il quale ai sensi dell'art.63 del DPR n.761/79 aveva il poteredovere di impartire istruzioni e direttive e verificarne la corretta esecuzione, eventualmente intervenendo personalmente (tanto più che era fisicamente presente in Pronto Soccorso), per rimediare ad eventuali errori.

Seguendo tali direttive esegetiche, la giurisprudenza è così giunta a sostenere un'applicazione maggiormente bilanciata del principio dell'affidamento reciproco tra i membri del gruppo di lavoro pluridisciplinare, assumendo una nozione di colpa professionale nell'attività medica d'équipe secondo la quale «i singoli medici devono ritenersi tenuti a rispondere per eventuali esiti lesivi ascrivibili ai propri collaboratori, quando avrebbero potuto accertare e segnalare detto evento al responsabile del gruppo, mediante l'impiego dell'ordinaria diligenza» [14].

L'obbligo di sorveglianza e di controllo dell'operato altrui del capo dell'équipe medica potrà sorgere anche nel caso di situazioni di emergenza o impreviste nelle quali le condotte dei partecipanti al gruppo di lavoro sono state inadatte rispetto alle capacità professionali richieste dal quadro clinico concreto in trattazione. In questo caso il compito principale del primario sarà quello di eliminare $o$ ridurre, se sollecitamente segnalati, tali inconvenienti, eventualmente anche mediante la sostituzione dei medici inadeguati con altri dotati di sufficiente preparazione.

In questi casi, infatti, «tenute in conto le anomalie comportamentali dei vari medici interessati al lavoro multidisciplinare - purché ovviamente segnalate al capo-équipe o da questi riconosciute direttamente - nonché la mancata corrispondenza delle stesse al grado di preparazione richiesto, insorgerà in capo al responsabile del gruppo di lavoro il preciso dovere di vanificare le condotte inadatte rispetto agli obiettivi terapeutici perseguiti, anche eventualmente mediante surroga dei medici artefici di simili atteggiamenti imprevisti» $[15,16]$.

Quanto al potere di sostituzione dei diversi componenti dell'équipe medica, si riporta ancora quanto sostenuto a tal proposito da Iadecola [9], il quale osserva che: «Esso va inteso come necessità di disporre previa ed opportuna ripartizione delle varie mansioni tra i componenti del gruppo, a garantire la copertura di tutte le esigenze che l'intervento comporta; quando ciò sia avvenuto, e sia stato correttamente percepito ed avviato dai collaboratori, e quando quindi la ripartizione stessa sia stata positivamente sperimentata, non pare necessario che il capo-équipe rinnovi le sue indicazioni prima di ogni trattamento o intervento, essendo sufficiente che egli riscontri la presenza di tutti i componenti dell'équipe e l'assenza tra essi di elementi nuovi». 
Si viene pertanto a delineare un vero e proprio obbligo di surroga da parte del responsabile dell'équipe medica - che trova le sue basi nel principio dell'affidamento reciproco - in tutti quei casi in cui una condotta connotata da negligenza, imprudenza o imperizia di uno o più sanitari interessati al lavoro di gruppo metta a repentaglio la riuscita del trattamento concretamente applicato al paziente. Ovviamente tale obbligo potrà scaturire anche dalla segnalazione operata dai singoli componenti dell'équipe, essendo tutti vincolati al dovere di rappresentare ogni eventuale inefficienza capace di poter compromettere il risultato di protocollo in corso di attuazione.

Nel quadro generale degli oneri gravanti su tutti i membri dell'équipe in virtù dell'affidamento reciproco, compito del responsabile del gruppo è in definitiva quello di svolgere un'incessante vigilanza sull'operato dei propri assistenti, onde poter tempestivamente contrastare ogni eventuale necessità o emergenza terapeutica emersa in sede di attuazione del trattamento.

Il dovere di sorveglianza incombente sul responsabile del gruppo di lavoro potrà pertanto essere compiutamente identificato mediante una pregnante verifica giurisdizionale delle modalità operative del trattamento concretamente applicato, facendo particolare attenzione ai momenti maggiormente delicati dello stesso e applicando più intenso rigore rispetto ai poteri di controllo sui membri dell'équipe virtualmente più esposti a imprevisti professionali. In questo senso la dottrina ha voluto altresì precisare, con riferimento al potere-dovere di controllo gravante sul responsabile dell'équipe medico-chirurgica, che: «in questo specifico caso accanto al criterio dell'affidamento, che continua ad ope- rare (ogni componente si deve anzitutto concentrare nel preciso compito che è tenuto a svolgere e gli altri gli si devono affidare, nel senso che devono essere sorretti dalla fiducia che si comporti con la dovuta attenzione, diligenza e professionalità), va considerato poi che il capo-équipe ha un dovere aggiuntivo di controllo e di supervigilanza sulle condotte di ciascuno e di tutti» [17].

\section{CONCLUSIONI}

Riassumendo, dunque, il dovere di sorveglianza gravante in capo al responsabile dell'équipe, implica innanzitutto una anticipata suddivisione degli incarichi tra i diversi componenti del gruppo medico di lavoro, necessaria per poter assicurare la copertura delle comuni esigenze terapeutiche $\mathrm{e}$ degli eventuali imprevisti che il trattamento possa comportare. Determinato il piano di lavoro necessario per la guarigione dell'assistito, non sarà più obbligatorio un pedissequo riscontro dei comportamenti posti in essere dai vari medici, essendo bastevole verificare la contemporanea presenza di tutti i membri del gruppo e la conseguente mancanza di componenti nuovi e inesperti.

La responsabilità colposa del capo-équipe per gli esiti nocivi del trattamento riconducibili all'operato negligente o imprudente altrui, sussisterà solamente qualora egli non intervenga mediante iniziative appropriate alle urgenze terapeutiche, determinando in tal guisa l'esito negativo della terapia medica.

\section{BIBLIOGRAFIA}

1. Buzzoni A. Medico e paziente. Le responsabilità civili e penali del medico e dell'equipe medica. Milano: Fag Edizioni, 2007

2. Corte di Cassazione, sez. IV, sentenza del 3 marzo 1988 "Grassi"

3. Mantovani F. Diritto penale. Padova: Cedam, 2002; p. 353

4. Fiandaca G, Musco E. Diritto penale. Parma: Zanichelli; p. 499

5. Mazzacuva N, Problemi attuali in materia di responsabilità penale del. Sanitario. Riv It Med Leg 1984; 6: 412

6. Belfiore E. Profili penali dell'attività medico-chirurgica in équipe. Arch Pen 1986; p. 297

7. Corte di cassazione, sez. IV, sentenza del 16 novembre 1990 "Rappezzo"

8. Cassazione penale, sez. IV, sentenza del 26 gennaio 2005. In: C.E.D. Cass., n. 231538

9. Iadecola G. I criteri della colpa nell'attività medica in équipe. Giur Merito 1997; 226

10. Corte di Cassazione, sez. IV, sentenza del 11 luglio 2002 "Volterrani"

11. Corte di Cassazione, sez. IV, sentenza del 12 luglio 2001 "Barese"

12. Corte di Cassazione, sez. IV, sentenza del 11 novembre 1994 "Presta"

13. Corte di Cassazione, sez. IV, sentenza del 22 settembre 1989 "Cipollato"

14. Cassazione penale, sez.IV, sentenza del 19 gennaio 1999 "Montaigner"

15. Cassazione penale, sez. IV, sentenza del 24 novembre 1999 "Pizza"

16. Cassazione penale, sez. IV, sentenza del 31 gennaio 1996 "Ferraro"

17. Vitale G. Responsabilità e rischi professionali. Cass pen 2000; 1051 